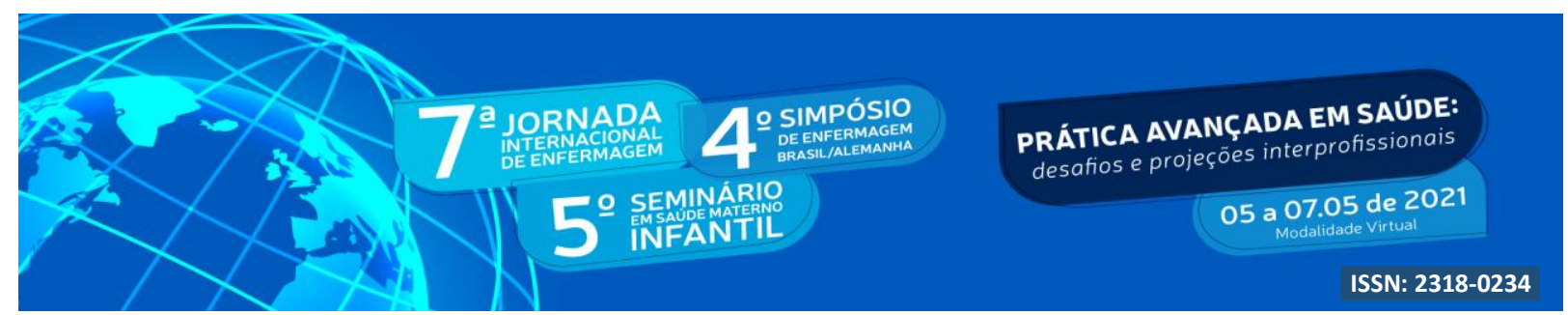

DOI: http://doi.org/10.48195/jie2021-138

\title{
MODELO EDUCATIVO PARA IMPLANTAÇÃO DE PROTOCOLO ERAS EM HOSPITAIS DE PEQUENA E MÉDIA COMPLEXIDADE
}

\section{Lucas Thomé Cavalheiro'; Mikaela Franco da Luz $^{2}$; Bruna Eduarda Foletto ${ }^{3}$; Anna Carolina Marchese dos Santos ${ }^{4 ;}$ Rosiane Filipin Rangel $^{5}$; Adalgiso Feijó Malaguez ${ }^{6}$}

\begin{abstract}
RESUMO
Objetivo: Estabelecer, com base na literatura moderna, um modelo educativo que facilite a implementação do protocolo ERAS com itens básicos ao paciente cirúrgico no período perioperatório em hospitais de médio e pequeno porte para auxiliar a equipe envolvida com o paciente cirúrgico a aplicarem todos os procedimentos de um protocolo multimodal de recuperação rápida, resultando em um melhor resultado pós-operatório de seus pacientes, com menores índices de complicação e tempo de internação. Metodologia: Foram incluídos para a implementação deste protocolo todos os profissionais de saúde envolvidos no preparo, na execução do procedimento e na recuperação do paciente no período perioperatório (médicos, enfermeiros, técnicos de enfermagem, nutricionistas e fisioterapeutas). Serão excluídos do protocolo todos os profissionais de saúde que não estão envolvidos com a recuperação perioperatória do paciente e aqueles que são destinados somente a cirurgias ambulatoriais. Resultados e discussões: Foram discutidas todas as ações que precisam ser realizadas antes da implementação, na implementação e após a implementação do protocolo. Além disso, foi proposto um modelo educativo para equipe de cuidados ao paciente perioperatório com base no modelo Team-based-learning (TBL). Todas essas ações resultam em maior adesão da equipe para implementar o protocolo, que resulta em melhores resultados na recuperação do paciente cirúrgico. Conclusão: Há uma mudança de paradigma em curso para otimizar manejo, reduzir custos de pacientes submetidos a cirurgias eletivas de baixa e média complexidade, também, com intenção de melhorar a satisfação dos pacientes em relação a
\end{abstract}

\footnotetext{
${ }^{1}$ Estudante do Curso de Medicina. Instituição de Origem: Universidade Franciscana E-mail: lucas.tcavalheiro@ufn.edu.br

${ }^{1}$ Estudante do Curso de Medicina. Instituição de Origem: Universidade Franciscana E-mail:. Instituição de Origem. E-mail: mikaelafranco6@gmail.com

${ }^{3}$ Estudante do Curso de Medicina. Instituição de Origem: Universidade Franciscana E-mail: b.folletto@ufn.edu.br

${ }^{4}$ estudante do Curso de Medicina. Instituição de Origem: Universidade Franciscana E-mail: anna.marchese@ufn.edu.br

${ }^{5}$ Enfermeira. Mestre e doutora em enfermagem pela universidade federal do Rio Grande. Docente da Universidade Franciscana. Coordenadora do programa de residência em enfermagem obstétrica. E-mail: rosiane@ufn.edu.br

${ }^{6}$ Docente curso Medicina. Instituição origem: Universidade Franciscana E-mail: amalaguez@ufn.edu.br
} 


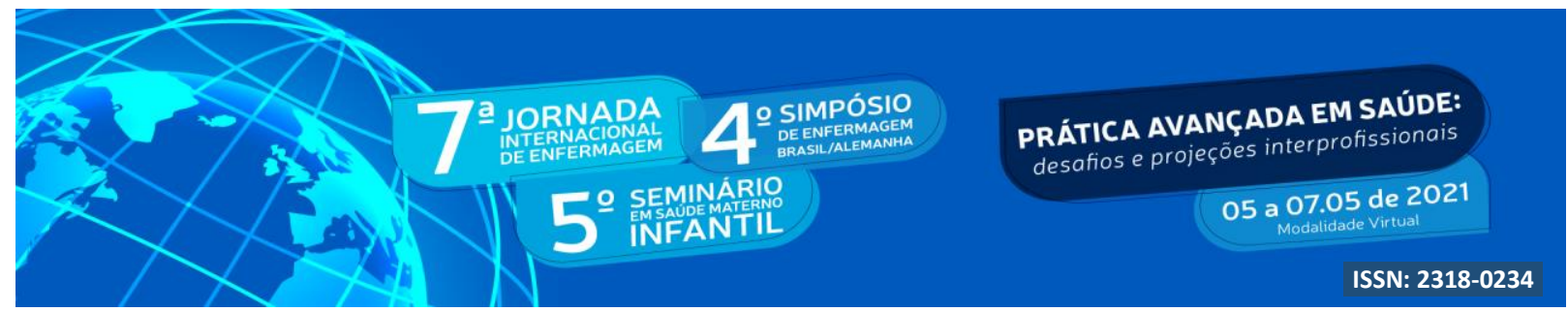

experiência do período perioperatório. A implantação destas mudanças, são necessárias e é preciso que se estabeleçam, para que aconteçam de fato na prática clínica-cirúrgica do dia a dia.

Palavras-chave: Assistência perioperatória, Nutrição cirúrgica, protocolo multimodal, Recuperação pós-operatória

\begin{abstract}
Objective: To establish, based on modern literature, an educational model that facilitates the implementation of the ERAS protocol with basic items for surgical patients in the perioperative period in medium and small hospitals to help the team involved with the surgical patient to apply all procedures of a multimodal protocol of rapid recovery, resulting in a better postoperative outcome of their patients , with lower complication rates and hospitalization time. Methodology: All health professionals involved in the preparation, execution of the procedure and recovery of the patient in the perioperative period (physicians, nurses, nursing technicians, nutritionists and physiotherapists) were included for the implementation of this protocol. All health professionals who are not involved with perioperative recovery of the patient and those who are intended only for outpatient surgeries will be excluded from the protocol. Results and discussions: All actions that need to be performed before implementation, implementation and after implementation of the protocol were discussed. In addition, an educational model was proposed for perioperative patient care team based on the Team-based-learning (TBL) model. All these actions result in greater team support to implement the protocol, which results in better results in the recovery of the surgical patient. Conclusion: There is a paradigm shift in progress to optimize management, reduce costs of patients undergoing low and medium complexity elective surgeries, also with the intention of improving patient satisfaction in relation to perioperative experience. The implementation of these changes are necessary and it is necessary to establish themselves, so that they actually happen in the clinical-surgical practice of daily life.
\end{abstract}

Key words: Perioperative care, Surgical nutrition, multimodal protocol, Postoperative recovery.

\title{
INTRODUÇÃO
}

Os programas de recuperação avançada são protocolos baseados em evidências, projetados para padronizar o atendimento médico, melhorar os resultados e reduzir os custos dos cuidados de saúde. Esses protocolos incluem técnicas baseadas em evidências para minimizar o trauma cirúrgico e a dor pós-operatória, reduzir complicações, melhorar os resultados e diminuir o tempo de internação hospitalar, ao mesmo tempo que agiliza a recuperação após procedimentos eletivos (KEHLET, WILMORE; 2008). O protocolo ERAS é um grande exemplo de um programa de recuperação avançada no período perioperatório, criado para atenuar a resposta ao estresse cirúrgico e reduzir a disfunção do órgão-alvo por meio de vias integradas pré-operatórias, intraoperatórias e pós-operatórias. Esse tipo de protocolo inclui 


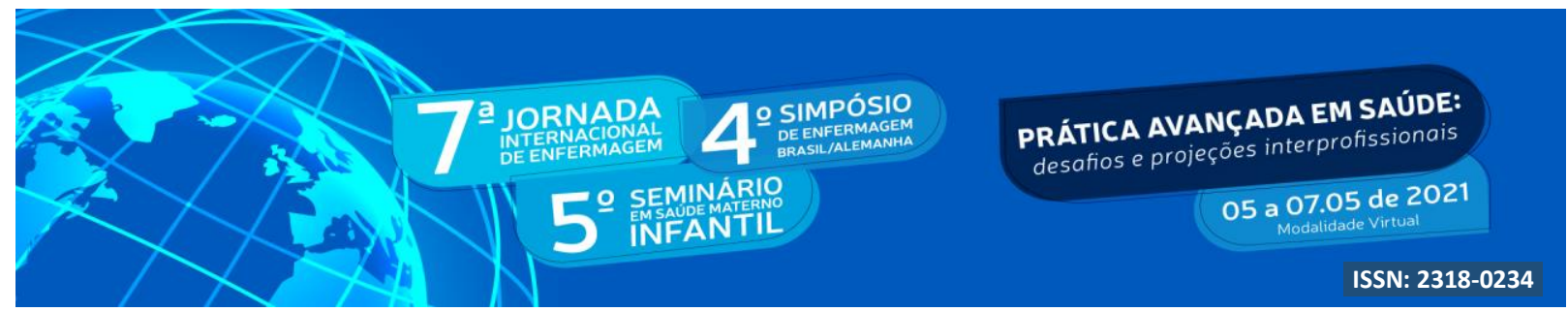

tipicamente 15 a 20 itens ou componentes combinados para formar uma via multimodal. Separadamente, os elementos individuais resultam em ganhos modestos, mas quando usados juntos de forma complementar, eles podem diminuir as respostas de estresse pós-operatório, reduzindo assim a duração do íleo pós-operatório, complicações cirúrgicas, dor incisional, tempo de recuperação e tempo de internação hospitalar. Assim, para desfrutar de todos esses benefícios é preciso que sua implementação seja bem-sucedida requirindo um esforço da equipe multidisciplinar, bem como a participação ativa do paciente. $\mathrm{O}$ início de tal caminho em tempo hábil e com boa relação custo-benefício pode ser um desafio logístico. Dito isso, sem implementação ativa, a difusão de alguns ou da maioria dos elementos das melhores práticas de recuperação aprimorada pode levar anos para ocorrer. Um esforço coordenado e multidisciplinar é necessário não apenas para a criação do programa, mas também para determinar os resultados que serão monitorados prospectivamente como parte de uma iniciativa de melhoria da qualidade (AGUILAR-NASCIMENTO; 2016).

Na prática clínica, existem três fatores que parecem ser os principais determinantes no dia a dia do profissional de saúde, são eles: o nível de evidência científica, o sistema de saúde junto com o contexto da prática profissional (acadêmico vs não acadêmico) e a presença de facilitadores de adesão à boa prática.

Os dois primeiros determinantes exigem, respectivamente, pesquisa em boa base de informação e hospitais com excelente qualidade de aparelhamento. São objetivos de médio ou longo prazo, pois exigem educação médica e investimentos na área de saúde. Por outro lado, os facilitadores locais de adesão às boas práticas (protocolos e diretrizes) são mecanismos implementados para assegurar que condutas reconhecidamente benéficas associadas à redução de morbidade e da mortalidade em bons estudos (randomizações, controles, revisões sistemáticas e metanálises) não sejam esquecidas pelos profissionais de saúde no dia a dia da prática clínica (AGUILAR-NASCIMENTO; 2016).

Um grande problema na subjetividade e na conduta médica, não fundamentada em protocolos, é o imaginário sobre o que se faz no dia a dia. Em uma pesquisa feita pelo projeto ACERTO com 12 cirurgiões do serviço de Cirurgia Geral do Hospital Universitário Júlio Muller (HUJM) sobre o uso de antibióticos em cirurgia, onze dos 12 cirurgiões afirmaram utilizar antibioticoprofilaxia em operações eletivas com ressecção intestinal. Ao mesmo tempo, 


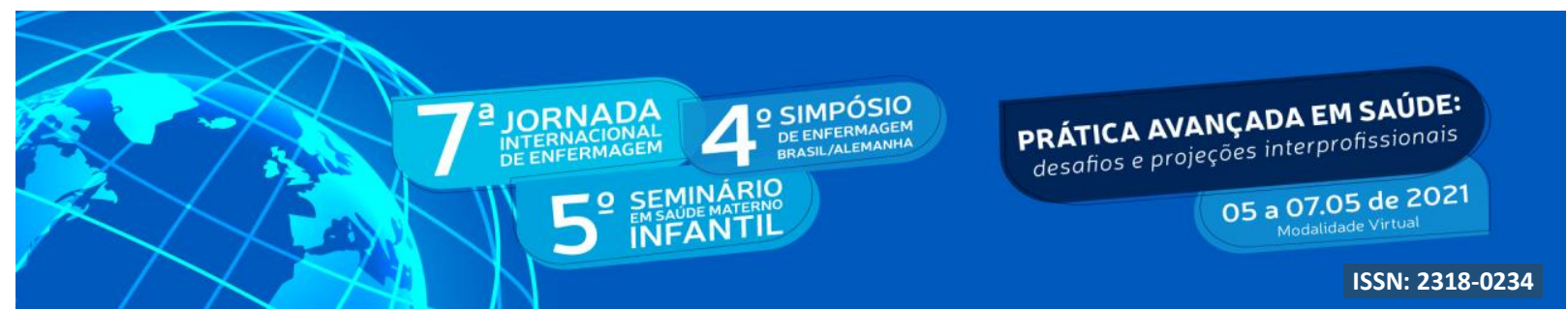

uma investigação prospectiva nas últimas 80 cirurgias com ressecção intestinal do serviço mostrou que a antibioticoprofilaxia por até $24 \mathrm{~h}$ no pós-operatório só foi utilizada em $10 \%$ dos pacientes. Esse fato, mostra a contradição entre o imaginário e a realidade em condutas médicas (AGUILAR-NASCIMENTO; 2016).

O protocolo ERAS, mostrou que é possível ter melhores resultados pós-operatórios, menor tempo de internação e redução de custos hospitalares com boas evidências científicas. Entretanto, percebemos baixa adesão entre os médicos dos serviços brasileiros. Por esse motivo, a implementação de auditorias e treinamento da equipe nos hospitais são de extrema importância. A auditoria é uma excelente ferramenta para aprimorar a qualidade de um serviço, enquanto o treinamento da equipe facilita a implementação do protocolo de forma bemsucedida (AGUILAR-NASCIMENTO; et al., 2008).

\section{METODOLOGIA}

Foram incluídos para a implementação deste protocolo todos os profissionais de saúde envolvidos no preparo, na execução do procedimento e na recuperação do paciente no período perioperatório (médicos, enfermeiros, técnicos de enfermagem, nutricionistas e fisioterapeutas). O grupo de pacientes que estes profissionais irão realizar a implementação do protocolo são os pacientes que serão submetidos a cirurgias de pequeno, médio e grande porte realizadas em bloco cirúrgico em hospitais de baixa e média complexidade.

Serão excluídos do protocolo todos os profissionais de saúde que não estão envolvidos com a recuperação perioperatória do paciente e aqueles que são destinados somente a cirurgias ambulatoriais.

\section{RESULTADO E DISCUSSÕES}

A execução bem-sucedida de um caminho de recuperação aprimorado envolve esforços coordenados e educação em vários departamentos e membros da equipe de saúde, também conhecidos como "partes interessadas". As partes interessadas incluem:

1. Pacientes, como destinatários das intervenções na via de recuperação aprimorada

2. Consultório do cirurgião, incluindo secretária e equipe de enfermagem

3. Cirurgião e equipe cirúrgica, incluindo provedores de prática avançada e estagiários 


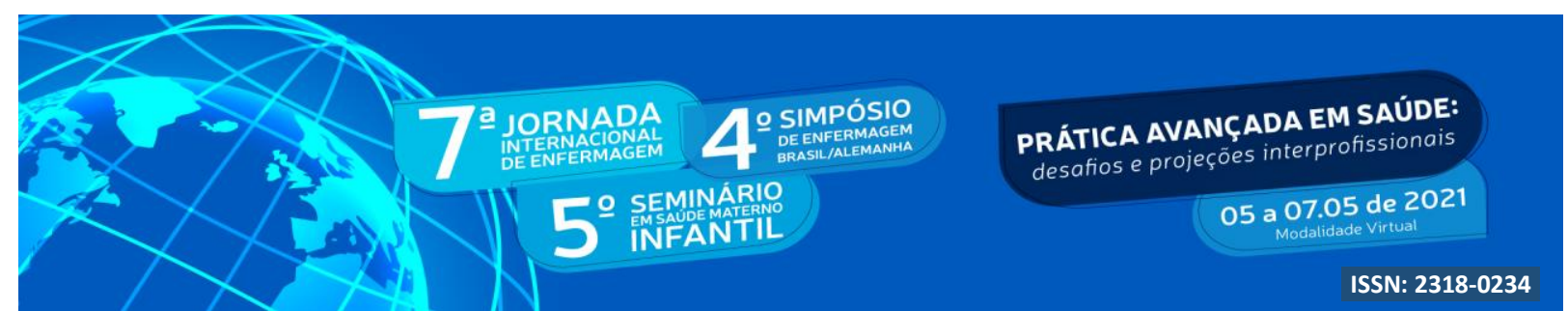

4. Anestesiologista e equipe de anestesiologia

5. Equipes de enfermagem pré-operatórias, intraoperatórias e pós-operatórias

6. Farmacêuticos e nutricionistas

7. Departamento de tecnologia da informação (TI)

Após a identificação de uma data de implementação, as etapas podem ser geralmente aplicadas da seguinte forma:

\section{Antes da implementação:}

1. Estabelecer representantes do Comitê Diretivo; identificar literatura e recursos do programa ERAS para disseminação dentro do departamento e comitê diretor.

2. Leia a literatura específica do ERAS.

3. Estabelecer consenso dentro do departamento sobre as populações-alvo do programa ERAS e elementos para adoção.

4. Reúna-se para obter consenso interdisciplinar sobre os elementos e executores ERAS.

5. Trabalho em conjuntos de pedidos com o departamento de TI; anunciar a iniciativa aos departamentos / partes interessadas.

6. Planeje os locais de educação pré-operatória, os materiais específicos de educação préoperatória do paciente e a carta pré-operatória.

7. Crie materiais educacionais formais para cirurgiões, anestesiologistas e enfermeiras contendo fundamentos e elementos ERAS.

8. Comece a enviar um novo documento pré-operatória; cirurgiões começam a agendar pacientes como "ERAS".

9. Envie lembretes de agendamento de pacientes cirúrgicos ERAS, pedido pré-operatório e datas de implementação para todos os interessados.

10. A educação pré-operatória do paciente começa / obtenha a bebida pré-operatória.

11. Realize o planejamento de ensino da equipe multidisciplinar. 


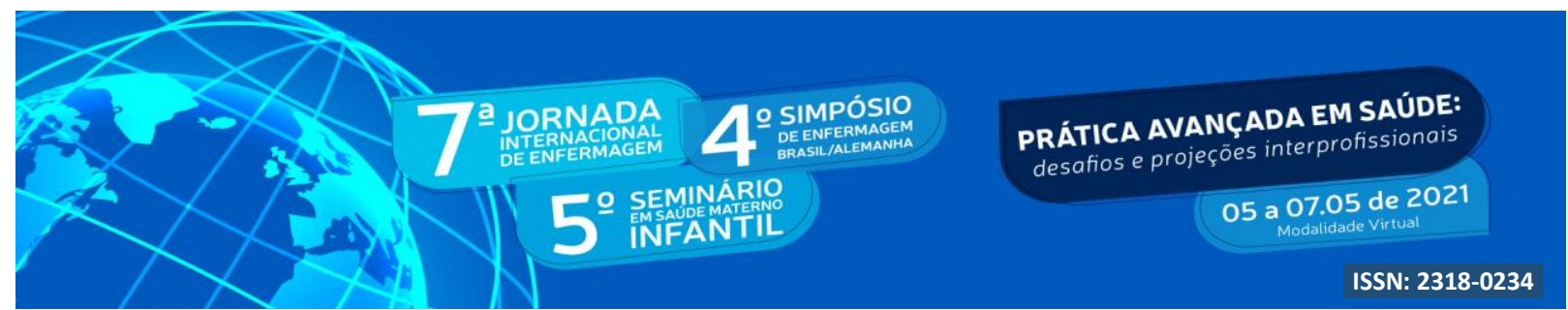

Um ponto importante a se considerar é qual a forma de ensino que será passada a equipe multidisciplinar, nesse protocolo a nossa metodologia será realizada com base no método de aprendizagem team-based-learning (TBL). Com isso, o ensino da equipe será feito em três etapas:

\section{Preparo}

Os integrantes da equipe multidisciplinar irão receber materiais informativos por meio de artigos (PubMed, Uptodate) e livros sobre o protocolo ERAS para realizar o estudo de forma individual uma semana antes da aula.

2. Garantia do preparo

Após os profissionais realizarem o estudo do tema, será revisado qual foi a capacidade de captação do conteúdo do grupo em três etapas: individual, equipes e apelação. No meio individual, o profissional da saúde realizara um teste avaliativo com questões múltipla escolha ou de verdadeiro ou falso. Após isso, as questões serão discutidas com toda a equipe para chegar em um consenso nas respostas. Caso houver alguma divergência com o gabarito, o profissional poderá escrever uma justificativa do motivo que não concorda com a resposta da questão.

3. Aplicação dos conceitos

O terceiro passo de ensino será a aplicação dos conceitos por meio da regra dos quatro ''S'" (Significado do problema, escolha do problema, aplicação do problema e respostas simultâneas).

Após todos os profissionais entenderem o conceito e a aplicabilidade do protocolo ERAS, cada um receberá sua função dentro dos itens do protocolo, a equipe multidisciplinar precisa estar coesa e alinhada para que o protocolo seja implementado com sucesso. As áreas da saúde que contemplam a equipe multidisciplinar são: anestesiologia, cirurgia, enfermagem, fisioterapia e nutrição.

\section{Implementação:}

A implementação do protocolo se divide em 3 partes, que são correspondentes aos períodos cirúrgicos que o paciente irá passar. 


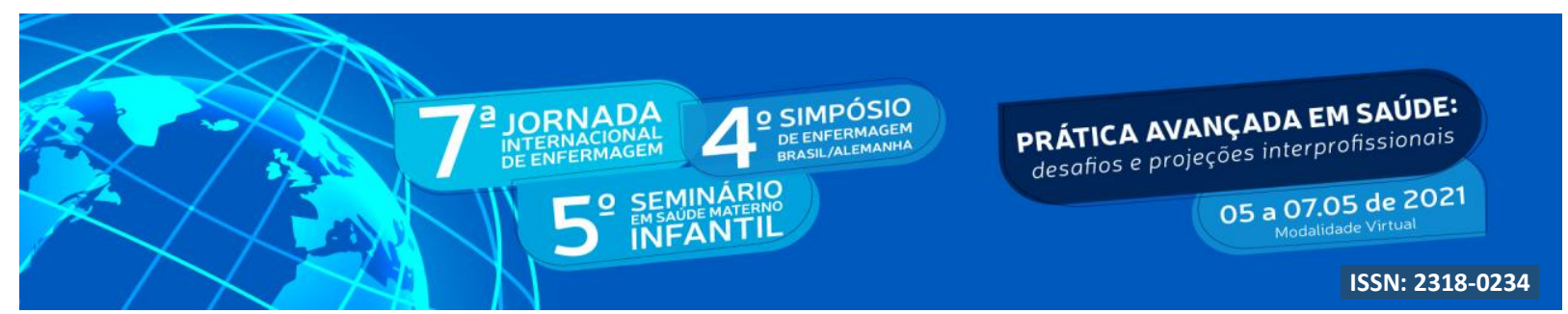

\section{Pré-operatório}

Educação do paciente: A educação pré-operatória define as expectativas do paciente para o processo de cirurgia e recuperação, o que pode, por sua vez, reduzir o medo, a fadiga e a dor, ao mesmo tempo que aumenta a alta. A educação eficaz do paciente pode assumir várias formas, incluindo acesso a módulos de atendimento on-line (AGUILAR-NASCIMENTO; 2016). Um método efetivo para uma boa orientação seria uma abordagem quádrupla para a educação do paciente, que inclui:

Consultório do cirurgião: O cirurgião discute com o paciente que ele estará em uma via de recuperação aprimorada para facilitar a recuperação cirúrgica e define a expectativa de alta na manhã do primeiro dia pós-operatório. $\mathrm{O}$ cirurgião também informa a paciente sobre o plano de acompanhamento pós-operatório, incluindo um telefonema pós-operatório do consultório do cirurgião dentro de dois a três dias após a alta hospitalar, bem como visitas pós-operatórias agendadas.

Consulta de avaliação pré-procedimento com a equipe de anestesiologia: Pacientes no caminho ERAS recebem uma sessão educacional de 15 minutos sobre o protocolo ERAS com uma enfermeira, além de passar por uma avaliação de risco pré-cirúrgica separada caso indicado clinicamente.

As informações fornecidas incluem o seguinte:

1. No pré-operatório, o paciente pode comer alimentos sólidos até a meia-noite da noite anterior à cirurgia.

2. Ela pode beber apenas líquidos claros até duas horas antes da cirurgia.

3. Ela receberá sabonete de clorexidina com o qual deverá se limpar na noite anterior e na manhã da cirurgia.

4. Se houver preocupação com desnutrição, ela receberá suplementos nutricionais préoperatórios (DROVER; et al.,2011).

5. Ela deve ser capaz de fazer uma dieta regular e deambular no início da noite da cirurgia (BAKHRU; et al., 2015).

6. Em seguida, ela deve deambular no corredor três vezes por dia.

7. O plano de alta na manhã após a cirurgia será revisto. 


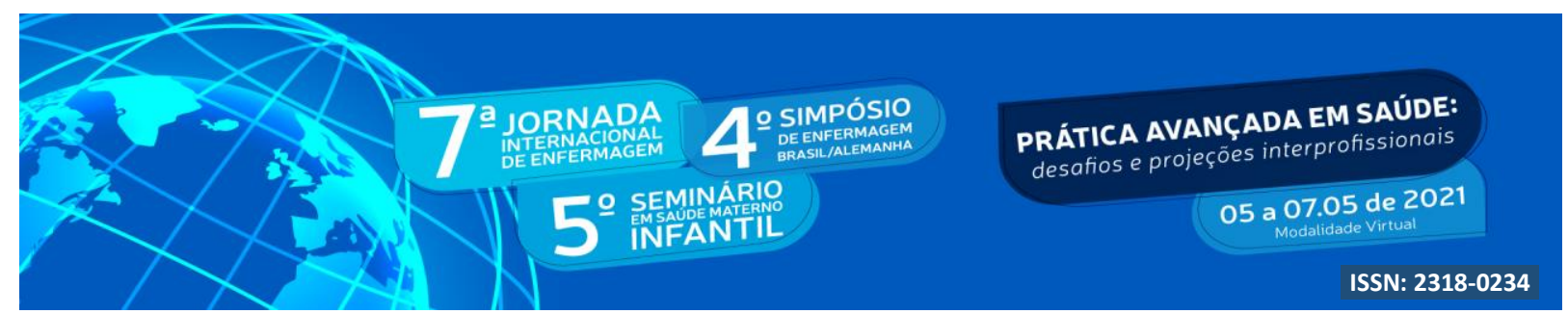

Intraoperatório

Analgesia e anestesia multimodal: Os elementos intraoperatórios dos protocolos ERAS são direcionados às equipes de anestesia e cirúrgica. A analgesia perioperatória multimodal é uma intervenção ERAS verdadeiramente interdisciplinar determinada por cirurgiões e anestesiologistas antes da cirurgia. As possíveis intervenções a serem consideradas incluem os tipos de analgesia pré-operatória, a abordagem mais rápida para a cirurgia pretendida e as oportunidades de minimizar a interrupção fisiológica (por exemplo, perda de sangue).

Sugerimos uma abordagem multimodal para analgesia preventiva com técnicas analgésicas regionais e drogas, como anti-inflamatórios não esteroidais (AINEs), paracetamol e gabapentinoides quando indicados, antes da indução da anestesia geral (Grau 2B) (AGUILARNASCIMENTO; 2016).

Intervenções específicas para a equipe cirúrgica: As intervenções ERAS específicas para a equipe cirúrgica incluem selecionar a via menos invasiva de cirurgia, determinar a necessidade e a duração do cateterismo vesical, evitar (ou remover no intraoperatório) tubos nasogástricos e minimizar o uso de drenos (AGUILAR-NASCIMENTO; 2016).

Acompanhamento pós-operatório: Além das visitas convencionais ao consultório do cirurgião no pós-operatório, um telefonema de acompanhamento logo após a chegada do paciente em casa pode diminuir a ansiedade do paciente, sendo útil para responder a perguntas, revisar instruções, fornecer garantias, identificar potenciais problemas ou complicações conforme surgirem, facilitar a transição do paciente do hospital para o ambiente doméstico e, potencialmente, reduzir chamadas evitáveis para o hospital e visitas ao departamento de. Nas instituições dos autores, esse telefonema pós-operatório é normalmente realizado por uma enfermeira ou médico dentro de um a dois dias após a alta. Esse conceito também está sendo cada vez mais aplicado a cirurgias ambulatoriais (LJUNGQVIST; SCOTT; FEARON; 2017).

Como o protocolo ERAS é composto de 23 itens espalhados dentro do período perioperatório, decidimos, no nosso protocolo, implementar apenas sete itens básicos do protocolo ERAS (Informação pré-operatória, jejum pré-operatório, terapia nutricional, alimentação imediata no pós-operatório, deambulação precoce, profilaxia para tromboembolismo venoso e prevenção de náuseas e vômitos) inicialmente para facilitar a 


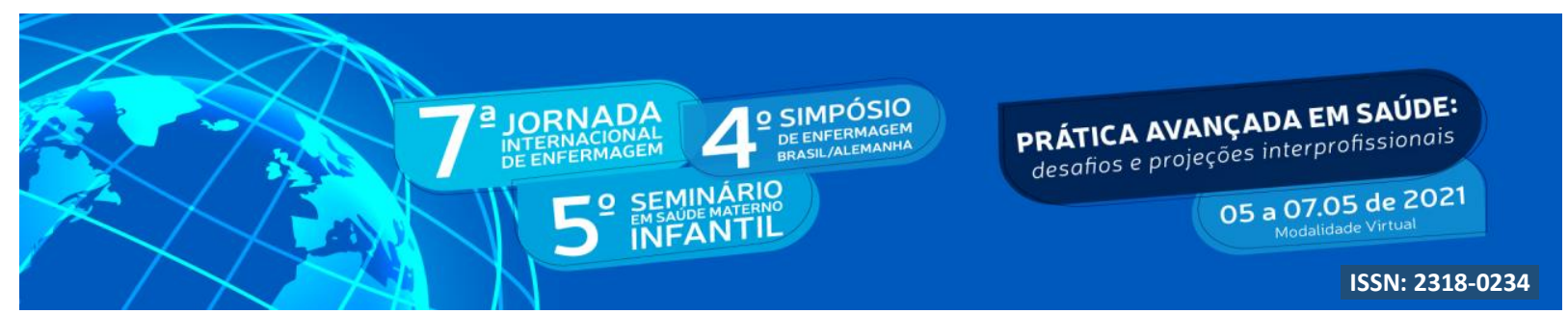

adesão da equipe. Após atingirmos números satisfatórios de aceitação os outros itens serão inseridos ao longo do tempo (AGUILAR-NASCIMENTO; et al., 2008).

\section{Após a implementação:}

1. Conduza conversas / educação clínica periódica

2. Realizar coleta / auditorias de dados de Melhoria da Qualidade

3. Adaptação e incorporação de novas evidências e melhores práticas

Como acontece com qualquer iniciativa de melhoria da qualidade, a implementação contínua e as auditorias clínicas são importantes. Os hospitais podem conduzir auditorias de melhoria de qualidade de forma independente; no entanto, a ERAS Society métricas de análise para facilitar o estudo da implementação e dos resultados clínicos (www.erassociety.org).

A seguir estão exemplos de métricas clínicas:

1. Tempo de internação

2. Custo de internação hospitalar

3. Uso de opioide perioperatório

4. Balanço de fluidos perioperatório

5. Escores de dor perioperatória

6. Dia pós-operatório de tolerância de dieta regular

7. Satisfação do paciente

\section{CONCLUSÃO}

Há uma mudança de paradigma em curso para otimizar manejo, reduzir custos de pacientes submetidos a cirurgias eletivas de baixa e média complexidade, também, com intenção de melhorar a satisfação dos pacientes em relação a experiência do período perioperatório. A implantação destas mudanças, são necessárias e é preciso que se estabeleçam, para que aconteçam de fato na prática clínica-cirúrgica do dia a dia. Este modelo educativo apresentado, contribui para um fácil entendimento e implementação do protocolo ERAS, sendo possível uma maior adesão da equipe cirúrgica e anestésica destes Hospitais 


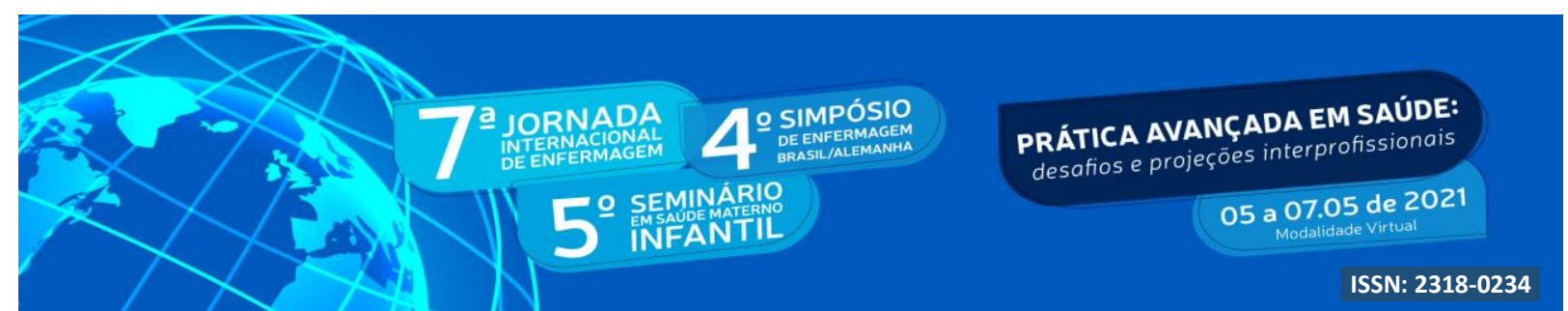

\section{REFERÊNCIAS}

AGUILAR-NASCIMENTO, J. E.; SALOMÃO, A.; CAPOROSSI, C.; SILVA, R.; SANTOS, T. P.; CARDOSO, E. A. Enhancing surgical recovery in Central-West Brazil: the ACERTO protocol results. European Journal of Clinical Nutrition. 2008.

AGUILAR-NASCIMENTO, José Eduardo. Acelerando a Recuperação Total Pósoperatória. 3 ed. Rio de Janeiro, Editora Rubio, 2016.

BAKHRU, R. N.; WIEBE, D. J.; MCWILLIAMS, D. J.; SPUHLER, V. J.; SCHWEICKERT, W. D. An environmental scan for early mobilization practices in U.S. ICUs. Critical Care Medicine. 2015.

DROVER, J. W.; DHALIWAL, R.; WEITZEL, L.; WISCHMEYER, P. E.; OCHOA, J. B.; HEYLAND, D. K. Perioperative use of arginine-supplemented diets: a systematic review of the evidence. Journal of the American College of Surgeons. 2011; 212(3): 385-99.

KEHLET, H.; WILMORE, D. W. Cuidado cirúrgico baseado em evidências e a evolução da cirurgia acelerada. Annals of Surgery; 2008; 248: 189

LERMAN, J. Surgical and patient factors involved in postoperative nausea and vomiting. British Journal of Anaesthesia. 1992.

LJUNGQVIST, O.; SCOTT, M.; FEARON, K. C. Recuperação aprimorada após a cirurgia: uma revisão. JAMA Surgery; 2017; 152: 292. 